\title{
The assertion-candidate and the meaning of $\operatorname{mood}$
}

\author{
Maria van der Schaar
}

Received: 18 August 2005 / Accepted: 12 June 2006 / Published online: 21 February 2007

(C) Springer Science+Business Media B.V. 2007

\begin{abstract}
The meaning of a declarative sentence and that of an interrogative sentence differ in their aspect of mood. A semantics of mood has to account for the differences in meaning between these sentences, and it also has to explain that sentences in different moods may have a common core. The meaning of the declarative mood is to be explained not in terms of actual force (contra Dummett), but in terms of potential force. The meaning of the declarative sentence (including its mood) is called the assertion-candidate, which is explained by what one must know in order to be entitled to utter the declarative with assertive force. Both a cognitive notion (knowledge) and a pragmatic notion (assertive force) are thus part of the explanation of the assertion-candidate. Davidson's criticism that such a theory is in need of an account of the distinction between standard and non-standard uses of the declarative is answered: without counter-indications an utterance of a declarative sentence is understood as having assertive force. The meaning of an interrogative sentence, the question-candidate, and that of the other sentence types can ultimately be explained in terms of their specific relations to the assertion-candidate. Martin-Löf's constructive type theory is used to show the philosophical relevance of a semantics of mood. The constructivist notion of proposition needs to be embedded in a theory of the assertion-candidate, which fulfils the offices of being the meaning of the declarative sentence, the content of judgement and assertion and the bearer of epistemic truth.
\end{abstract}

Keywords Assertion · Judgement - Meaning of mood ·

(Mathematical) Constructivism

\footnotetext{
M. van der Schaar $(\varangle)$

Faculty of Philosophy, Leiden University,

P.O. Box 9515, 2300RA Leiden,

The Netherlands

e-mail: m.v.d.schaar@let.leidenuniv.nl
} 


\section{Introduction}

One may note a difference in each of the following sentences:

(1) The door is closed.

(2) Is the door closed?

(3) Close the door!

Linguists classify these sentences as belonging to different types (Huddleston \& Pullum, 2002, p. 853): (1) is a declarative sentence; (2) an interrogative sentence; and (3) an imperative sentence. The grammatical difference between each of these sentences can be read from the inflexion of the verb and the word-order. The question that is addressed to in this paper is whether there is also a difference in meaning between these sentences.

Michael Dummett and Donald Davidson each have given an affirmative answer to this question, and have proposed a theory of meaning of what they call mood. Mood is what distinguishes each of the above-mentioned sentences: the first sentence is said to be in the 'indicative' mood; the second in the interrogative mood; and the third in the imperative mood. I will follow them in their terminology, except that I will speak of 'declarative mood' instead of 'indicative mood', because the term 'indicative mood' is already in use to classify the inflexions of the verb, along with such terms as 'subjunctive' and 'conjunctive mood' (In this sense, the verbs of sentence (1) and (2) are both in the indicative mood).

Dummett and Davidson explain the meaning of mood in terms of force. Force pertains to the use made of an utterance (or occurrence) of a sentence within a linguistic-communicative situation; force is a characteristic of uses of free-standing (occurrences of) sentences only, not of (occurrences of) sentence-clauses. At first sight, there seems to be a relation between the declarative sentence, a sentence in the declarative mood, and the fact that we may use an utterance of the declarative to make an assertion, that is, that we may use it with assertive force. A similar relation there seems to be between a sentence in the interrogative mood and the fact that an utterance of the interrogative sentence may be used to ask a question, that is, with interrogative force. And there also seems to be such a relation between the imperative mood and the force of command.

According to Dummett, a theory of meaning includes not only a theory of reference and sense, but also a theory of force (Dummett, 1976, p. 40). A theory of force is closely related to a theory of mood, because mood is, according to Dummett, a prima facie sign of force. According to Davidson, there cannot be such a direct relation between mood and force. Occurrences of each of the above-mentioned sentences may be used with all kinds of force. We often use a declarative sentence to give an order, or we make an assertion by uttering an interrogative sentence.

I will argue that Davidson's criticism of Dummett is partly correct: the relation between mood and force is less direct than Dummett thinks. But it will also be argued that Davidson's account of mood is essentially incomplete: an account of the declarative mood and of the mood of open interrogatives ('Which door is open?') is missing. By making a distinction between actual and potential force it is possible to give an account of mood that answers both forms of criticism. The philosophical importance of a semantics of mood, in particular the declarative mood, will become clear, when one understands that a notion of judgement is needed that is more complex than the standard Fregean one, and that a distinction between semantic and epistemic truth 
needs to be made. Although these topics are not exclusive to Martin-Löf's constructive type theory, they are more developed there than anywhere else, and I will use this theory to show the philosophical relevance of a semantics for mood.

\section{Dummett, Davidson and Mood}

According to Dummett, the meaning of mood is not to be explained in terms of the internal state of the speaker. Essential to the utterance of a declarative sentence is not that it expresses a certain belief. The presence of the declarative mood in the utterance of a sentence is, rather, a prima facie indication that an assertion is being made (Dummett, 1993, p. 207). And the utterance of a sentence in the imperative mood in itself constitutes the giving of a command, unless special circumstances divest the utterance of its usual significance (Dummett, 1973, p. 302). Dummett admits that an utterance of the declarative sentence may be used to ask a question, but this fact does not have any influence on the force of the utterance. In order to explain this he makes a distinction between the force and the point of an utterance. An utterance of a free-standing declarative always has assertive force; the point of the utterance may be different, though, for example to ask a question. What a speaker actually says, the force of his utterance, is determined by the conventions of a language, whereas the point of the utterance is determined by the intentions of the speaker (Dummett, 1993 , p. 209, 210). To give a theory of meaning is to give a systematic account of such conventions; a theory of meaning cannot and need not give an account of the intentions of the speaker. Dummett's distinction between the force and the point of an utterance cannot account for all the different uses we make of free-standing declaratives. When a speaker utters a declarative to give an example, the utterance does not have assertive force. Besides, Dummett does not give an account of declaratives that are not free-standing. What is the meaning of the declarative in case it is used as antecedent of a conditional sentence? It seems that it cannot be given in terms of assertive force, for the antecedent is not uttered with any force.

According to Davidson, Dummett's theory is in need of a non-circular account of the distinction between standard and non-standard uses of mood. 'Normal' or 'standard' use cannot mean serious use, for a command that is given by an utterance of a declarative sentence is not less serious than a command given by an utterance of the imperative. 'Normal' cannot mean statistically more frequent, for it is doubtful that most declaratives are uttered with assertive force (Davidson, 1979, p. 111). And an account of the distinction in terms of convention does not work, either. Nothing can be a conventional sign of sincere assertion, for every good actor would make use of such a sign. If the relation between mood and force were conventional in such a direct way, there would not so often be a difference between mood and 'standard' force: "You can't use a convention by breaking it" (Davidson, 1984, p. 275).

Davidson gives an account of the meaning of mood in terms of force without going beyond a theory of reference. In order to explain the meaning of mood in terms of truth-conditions, Davidson analyses an utterance of the interrogative sentence 'Does it snow?' as 'My next utterance is interrogatival in force. It snows.' The utterance of the interrogative sentence is decomposed into two distinct speech acts: the utterance of a mood-setter, which refers to the force of the original utterance, and the utterance of a declarative ('indicative') sentence. If the original interrogative is not used for asking a question, the sentence containing the mood-setter is false. The meaning of 
the composing sentences is completely given in terms of truth-conditions (Davidson, 1979, pp. 119-120); mood is explained in terms of reference. The utterance of the declarative 'It snows.' is supposed to be neutral: it should not be put forward with assertive force, on pain of circularity.

Davidson's semantic analysis of the declarative sentence contains a problem. Either the declarative sentence needs to be given a double semantic explanation: as having declarative mood and as neutral sentence or the declarative sentence is explained only as neutral sentence. As Davidson would not allow for two explanations of the same sentence type, the declarative sentence is considered to be neutral, and the way in which the declarative mood differs from the other moods is left unexplained. Davidson's theory of the meaning of mood is essentially incomplete: the meaning of the declarative mood is missing. By using the declarative as neutral sentence, Davidson is not able to explain the intuition that declarative, interrogative and imperative sentences differ from each other in a similar way. ${ }^{1}$

An important argument for Davidson's explanation of the declarative as neutral sentence is that the declarative sentence has to function as antecedent in conditional sentences. In such cases the declarative is not put forward with assertive force. Because the declarative may as well be used for commands and questions, Davidson concludes: "Indicatives we may as well leave alone, since we have found no intelligible use for an assertion sign." (Davidson, 1979, p. 119). The neutral declarative can thus be used to explain our intuition that the different sentence types (1), (2) and (3) have something in common.

Davidson's theory of the meaning of mood is also incomplete in another way. How are we to analyse an utterance of the open interrogative sentence 'Where does it snow?'. The latter cannot be analyzed as 'My next utterance is interrogative in force. It snows (somewhere).', for that counts already as an analysis of (an utterance of) the sentence 'Does it snow (somewhere)?'. There is not a complete neutral declarative sentence that corresponds to the open interrogative. The semantics for open interrogatives forms a problem for any theory of mood. For Davidson this problem seems to be more fundamental, because his semantics is not to go beyond a theory of reference. What reference could an incomplete neutral sentence have? Certainly, that reference cannot be given in terms of truth-conditions.

A theory of the meaning of mood (1) has to give an account of our intuition that there is a common core for the different sentence types, and (1a) of our intuition that the open interrogative is deviant in this respect. It also (2) has to explain in what sense each of the sentence types, including (2a) the declarative, differs from any of the others. If a theory of mood is able to do all this, it may be called complete. Further, the theory has to explain (3) our intuition that there is a special relation between mood and a corresponding force, for example, between the imperative mood and the force of command. At the same time, it (4) has to give an explanation of deviant uses of the different sentence types. It has to explain that (4a) we may use the different moods with all kinds of force. It also (4b) has to give an explanation of weakened force: an imperative sentence may be used not only to give a command, but also to make a request, in case there is no authority of the speaker over the hearer. Further, (4c) the

\footnotetext{
1 The term 'declarative sentence' that I have chosen may seem less apt for a neutral sentence than Davidson's 'indicative sentence', because the meaning of 'declarative' is standardly explained in terms of assertion (cf. the Oxford English Dictionary). The OED gives as meaning of 'indicative' in so far as it applies to sentences: "Of a form of statement: Having the verb in the indicative mood; assertive of objective fact". In this respect the terms 'declarative' and 'indicative' do not differ.
} 
theory has to explain the special use that is made of the different sentence types on stage. And the theory has to give an account of the absence of force in (4d) examples and (4e) quotations. In the King James Bible one may find the sentence 'There is no God.' (Psalm 53: 2). The bible has not asserted that there is no God, for the sentence is preceded by the sentence 'The fool hath said in his heart.' And if the teacher writes down on the black-board the declarative sentence 'Every boy loves a girl' in a logic class room, the sentence is not uttered with assertive force. The same may happen to sentences of any type in an article on mood. Last, but not least, the theory has to give an explanation of (4f) the fact that the moods can appear as subordinate clauses of conditional and disjunctive sentences. The problem is not restricted to declarative sentences, for we also use non-declarative sentences as subordinate clauses. Neither Davidson nor Dummett have acknowledged this point. Dummett explicitly says that "the interrogative and imperative moods cannot figure in subordinate clauses" (Dummett, 1993, p. 204). When an utterance of the sentence 'Love the Lord or leave his land.' is used with the force of command, the utterance of each of the subordinate clauses, though in the imperative mood, does not have any force.

\section{The sign of assertion and the meaning of the declarative mood}

Is it possible to explain the intuition that there is a special relation between the declarative mood and assertive force? The full stop at the end of a sentence together with a capital at the beginning is a sign that the sentence is free-standing and can be used with a certain force. Only if we can already make a distinction between standard and non-standard uses of the declarative, we can take the full stop as a sign that an assertion has been made. If the declarative is used as a subordinate clause in a conditional sentence, then the declarative is preceded by 'If', and there is thus a clear sign in the context of the utterance that it does not have assertive force. In general, if an utterance of the declarative is not to be taken as an assertion, the context has to provide indications that the assertive force is cancelled.

Further, in the explanation of declarative mood in terms of assertive force, it is not relevant whether the speaker meant his utterance to have assertive force, but whether the hearer understands the utterance as an assertion. Suppose that someone says to you 'I see you blushing with shame.', whereupon you try to hide your face, and he then adds 'I didn't mean it. I was only joking.' You rightly took his utterance in the first instance as an assertion. By taking the point of view of the hearer/reader as crucial, and by asking for a sign that cancels assertive force, it is possible to relate declarative mood to assertive force: without counter-indications an utterance of a declarative sentence is understood as having assertive force. Dummett is right: there is a characteristic or typical use of the declarative, namely to utter it with assertive force.

The sign that cancels the assertive force may be compared to what is traditionally called a 'modifying adjective', which term stands in contrast to that of 'attributive adjective'. If an attributive adjective precedes a general term in a singular, atomic sentence, one may validly draw the conclusion that the subject falls under (the concept denoted by) the general term. From the sentence 'He has a German pistol.', in which 'German' is used attributively, we may validly draw the conclusion 'He has a pistol.'. If a modifying adjective precedes a general term, for example 'sham' in 'He has a sham pistol.', we cannot validly draw the conclusion that he 
has a pistol. The meaning of the modified term 'pistol' in 'sham pistol' is secondary in the order of explanation to the meaning of the non-modified term 'pistol'. One can understand the meaning of the expression 'false friend', only if one understands the meaning of the non-modified term 'friend'. The meaning of the former is systematically related to, and one-sidedly dependent upon the meaning of the latter. The appearance of a false friend is the same as that of a friend, but the false friend is deprived of something that is essential to a friend-that it is someone you can trust.

The operation of modification can be extended from adjectives to contexts in general, and from the meaning to the use of an expression. If an utterance of the declarative is preceded by the term 'If', the assertive force of the utterance is cancelled. In this sense the term 'If' modifies the typical use of the declarative. A similar extension of the operation of modification can be found in Husserl's fifth Logical Investigation. The quality of a judgement, its act-character, may be modified into an act of mere entertainment: instead of judging that it snows, one merely thinks that it snows. Because the quality, the act-character, is changed, the operation is called 'qualitative modification' (Husserl, 1901, V, Sect. 38; cf. Stepanians, 1998, Ch. 10). Husserl stresses the point that the operation of qualitative modification cannot be iterated (idem, Sect. 39). Exactly the same is often said of the assertion-sign, and in this sense both differ, for example, from the operation of negation. It should be noted that the term 'operation' is used here to denote a qualification of the act; it thus differs essentially from the term used for negation, which operates on the propositional content.

Frege's assertion-sign (Urteilsstrich) makes sense, from a psychological point of view. We first understand a proposition (das Fassen des Gedankens, Frege, 1918, p. 62), and then judge it to be true (die Anerkennung der Wahrheit des Gedankens). From a conceptual point of view, though, the assertion-sign is superfluous, because a free-standing occurrence of the declarative sentence is already understood as having assertive force. Wittgenstein was right, when he said that Frege's assertion sign is logically meaningless (Tractatus 4.442). Inspired by Wittgenstein, Erik Stenius has introduced a sign that cancels assertive force. If a declarative sentence $p$ is not asserted, it is put within brackets: $(p) .^{2}$

If it is true, as Dummett says, that questions about meaning are best interpreted as questions about understanding (Dummett, 1976, p. 35), what is it to understand a declarative sentence? Understanding a declarative is understanding what kind of speech act is typically made by an utterance of that sentence, which is the speech act of assertion. Further, it involves understanding what kind of assertion would thus be made, and under what condition one is entitled to make the assertion in question. Understanding a declarative does not imply that one understands what actual use is made of the sentence. The meaning of a declarative sentence-including its declarative mood-is to be explained not in terms of actual force, but in terms of potential force. It is thus possible to give the same meaning to the declarative whether it is used as a free-standing sentence or as a subordinate clause.

Each expresses what may be called an assertion-candidate. The assertion-candidate expressed by a declarative sentence will be explained in terms of the act of assertion

2 Cf. Stenius (1960, p. 163; 1967, pp. 254-255). Erik Stenius was one of the first, together with Hare, to give a theory of mood-though his is a theory of what he calls 'semantic mood', that is, of force. Cf. Stenius (1960, p. 168; 1967, pp. 256-257).

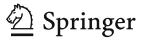


that an utterance of the declarative is typically understood to have, and in terms of the condition under which one is entitled to make the assertion in question. Before giving such an explanation, it is necessary to distinguish the assertion-candidate from related notions: that of assertion, proposition and declarative sentence. It is not possible to give a philosophically neutral explanation of these notions. Independent of the philosophical commitment that is made, it should become clear that the assertioncandidate is distinct from each of these notions.

When someone uses an occurrence of the declarative sentence 'Snow is white' for making an assertion, an interlocutor is entitled to ask: 'How do you know?'. If the asserter is not able to give grounds for his assertion, he has to withdraw it. ${ }^{3}$ To assert is to claim that one is entitled to make the assertion in question, and one is entitled to make that assertion if and only if one is epistemically justified to do so. The condition under which one is entitled to assert is cognitive and internalist in nature.

Judgement and assertion do not differ essentially. The internal notion of judgement can be explained in terms of the external notion of assertion: judgement is the interiorization of assertion (cf. Dummett, 1973, p. 363). The notion of judgement is thus cognitive to the extent that the notion of assertion is. Furthermore, 'assertion' and 'judgement' exhibit an ambiguity between the act of asserting or judging and its prod$u c t$, the assertion that is asserted in the act, the assertion or the judgement made. The product of an act is not caused by the act; the relation between act and product is not a natural one. There is an internal relation between act and product, such that the (complete) act necessarily gives rise to its product. ${ }^{4}$ Whereas mental acts and speech acts have a momentaneous existence in space and time, their product is an abstract object, not having such an existence, but being valid in the sense in which we call a verdict (product) of a judge in court 'valid' any moment after he has convicted the suspect.

Both act and product of assertion need to be distinguished from the notion of proposition. In an assertion made by means of an utterance of the declarative 'Snow is white' the proposition that snow is white is asserted to be true. The assertion made by means of an occurrence of the declarative 'Snow is white' has declarative form (Snow is white), and is dependent upon the act of asserting, whereas the proposition that snow is white has the form of a that-clause, and is not dependent upon such an act. A speech act, or its mental counterpart, is a deed, and its product is that which is done; the proposition, in contrast, is a meaning-entity. The assertion made by an utterance of the declarative 'Snow is white' and the question asked by an utterance of the interrogative sentence 'Is snow white?' have the same proposition as (part of their) content, whereas the two products differ essentially, being an assertion made and a question asked, respectively.

The assertion-candidate is different from both act and product of assertion. The assertion-candidate need not be actually asserted; it is what can be asserted. The mere candidate differs from the assertion made in that it has no force; the assertion-product is the asserted assertion-candidate. With respect to any declarative, and the assertion-candidate expressed, one may always ask: 'Is this correct?', but only with respect to an asserted declarative is one entitled to ask: 'How do you know?'. Although both assertion-candidate and assertion-product are abstract entities, only

3 The original meaning of 'to assert', like the meaning of 'to judge', is legal: "to put one's hand on the head of a slave, either to set him free or claim him for servitude" (OED; cf. the Latin assero). 'To declare' (used in 'declarative') has a cognitive origin; Lat. 'declaro' means I make clear. These terms originally involve a claim for which a (legal or cognitive) entitlement is needed.

${ }^{4}$ More on the distinction between act and product can be found in van der Schaar (2006). 
the assertion-candidate is a meaning-entity. Whereas the assertion-product is essentially the result of someone's act of assertion, this is not the case for the assertioncandidate. The assertion-candidate is the assertion stripped of its assertive force; the term 'candidate' is a modifying term.

The assertion-candidate differs also from the proposition. The assertion-candidate snow is white and the proposition that snow is white are closely related, but they differ as far as their structure is concerned: the assertion-candidate has declarative form, whereas the proposition has a that-structure. Snow is white and that snow is white is true are identical as assertion-candidates, because the same assertion is made by an utterance of the corresponding declaratives. One might thus say that the assertioncandidate is the proposition together with the is true element.

Finally, one may ask to what extent the assertion-candidate differs from the declarative sentence (as type). If one understands a sentence not in purely physical terms, but as a linguistic entity, it essentially expresses a meaning. Expression and meaning are dependent upon each other. Speaking of the assertion-candidate is speaking of this linguistic entity in its meaning-aspect; speaking of the declarative sentence one is speaking of the linguistic entity in its formal aspect. If the declarative sentence contains indexical terms, it is incomplete in relation to the assertion-candidate. The declarative 'I am here' expresses a new assertion-candidate each time it is uttered by another person, another place, or another time. Leaving the problem of indexicals for another occasion, for now one may say that in such cases an assertion-candidate is expressed by the declarative sentence together with elements of the context of utterance, such as speaker, time and place.

Traditionally, the ambiguities of the term 'judgement' made it possible to take account of the assertion-candidate under that term. ${ }^{5}$ The distinction between judgement and judgement-candidate, which is identical with the assertion-candidate, was generally acknowledged in the middle ages. This was facilitated by their terminology. What they called 'proposition' has the declarative form: snow is white. If the 'proposition' is asserted, it is called propositio iudicativa; if it is merely entertained, it is called propositio apprehensiva (Ockham, Quodlibetal Questions, Quodlibet 5, Questio 6; the distinction was common knowledge, see Nuchelmans, 1980, p. 27, 89). Irrespective of whether an assertion is made, the assertion-candidate, the enuntiabile, is that which is assertible (Nuchelmans, 1973, p. 169). It is assertion-apt, because it has the proper form for being asserted. Whether the assertion-candidate is also assertible in the sense of "worthy of being asserted" $(O E D)$ depends on its being correct or not.

The notion of assertion-candidate also has a counterpart within phenomenology. The assertion-candidate is what Husserl called the judgment in a logical sense, which is an act-species or ideal meaning that can be (but need not be) instantiated by an act of judgement (Husserl, 1901, I, Sect. 30-34). The act of judgement consists of two dependent parts, two moments: quality and matter. The distinction between quality and matter is comparable to the now common distinction between force and content of an act. The quality and matter of an act make up the intentional or meaningessence of the act (Husserl, 1901, V, Sect. 21). The ideal judgement, an act-species, is an idealization of both elements of the meaning-essence of the act. Although the ideal

\footnotetext{
5 The notion is old, but it led a curiously neglected life. It may be found in different forms in several writers. It can be found in Martin-Löf (1998), where the term 'judgement' is used, and in his (1996), where he uses the term 'enunciation'. It can also be found in Sundholm (1999), who uses the term 'statement'. A similar notion and term ('candidate judgment') can also be found in Siegel (2004, p. 601, 607). The term 'assertion-candidate' was suggested to me by Göran Sundholm.
}

Springer 
judgement is not an act itself, part of it corresponds to the matter and part of it to the quality of the act that instantiates it. Because it also contains the latter, the (ideal) meaning of a declarative sentence corresponds to the assertion-candidate rather than to the modern notion of proposition.

\section{Constructivism and the assertion-candidate}

The relevance of the notion of assertion-candidate cannot be given by merely distinguishing it from related notions, as has been done above; its raison d'être is dependent upon its philosophical need. Such a need, though, cannot exist independently of any philosophical framework.

Logical theory knows various offices that have to be filled by entities of certain kinds, of which the most important three are:

- the meaning of a sentence;

- the bearer of truth and falsity;

- the content of thoughts, assertions, judgements and closed questions. ${ }^{6}$

In each case a kind of entity is called for. In a Fregean theory, the Gedanke or proposition that $S$ serves in all three roles. For Frege, the primary role of the proposition is being the bearer of truth and falsity. The proposition also fulfils the role of content of judgements and closed questions. When someone asks the question 'Is snow white?' and gets the answer 'Snow is white.', there is something which makes this an answer to the former question: both have the same content, namely the proposition that snow is white (Frege, 1918, p. 62). Further, the proposition is considered to be the meaning (Sinn) of a declarative sentence.

Fregean propositions are independent of language and a judging subject, and it is difficult to explain the meaning of mood within the frame-work of Frege's logical realism (In section 5, I will show that in 1892, Frege had something to say on the meaning of mood). Without a language-using subject there cannot be any force or speech act, and the meaning of mood is to be explained in such terms. Probably, a phenomenological or pragmatic framework can be used to show the philosophical relevance of the assertion-candidate, because of the importance that is given to the act of a cognitive and language-using subject. My choice, though, is on Martin-Löf's constructive type theory. The assertion-candidate expressed by a sentence $S$ is to be explained in terms of the condition under which one is entitled to assert that $S$, and one may expect constructivism to say something on that cognitive condition. Further, type theory acknowledges different judgemental forms which cannot be explained purely in terms of propositions and the assertion-sign. Martin Löf's constructive type theory, which acknowledges different roles of truth, may also help to clarify a distinction that Erik Stenius has made regarding truth: there is truth pertaining to propositions (descriptive truth), and there is truth pertaining to sentences in the declarative mood if someone plays the language-game of the declarative mood (the game of assertion) correctly (modal truth, Stenius 1960, p. 168). Within constructivism some use is made

6 The question whether there is a fourth office, namely the (identical, intentional) object to which our acts are directed, and what type of entity is to fulfil this role, I leave for another occasion. Van der Schaar (2003) and Martin-Löf, in a lecture held in Geneva in December 2003, address the question what the object is of the so-called propositional attitudes; neither of them makes a distinction between the object and the content of the act. 
of phenomenological ideas. This was done in the thirties by Arend Heyting and Oskar Becker regarding Husserl's notion of fulfilment. Martin-Löf also makes use of the phenomenological tradition: Brentano's explanation of correctness in terms of the evident judgement is, for example, of importance to his framework. ${ }^{7}$ Before asking the question what kind of entity fulfils the above-mentioned offices within constructive type theory, I will first explain some fundamental notions of that framework, namely the notions of judgement, judgemental correctness and knowledge.

The notion of judgemental act is a primitive one (Martin-Löf, 1991, p. 144). The notion is also cognitive, according to Martin-Löf. A judgemental act is an act of getting to know, and the judgement that is a product of that act is (a piece of) knowledge. For Martin-Löf, the distinction between act and product of judgement that was introduced in the former section is thus crucial to his explanation of knowledge. The judgemental act through which knowledge is obtained may be an act of inference (a proof-act), which results in a proven theorem or it may be an act of understanding, which results in an axiom (Martin-Löf, 1987, p. 417; 1991, p. 146). Instead of saying that a proven theorem is the result of an act of inference, one may also say that the proven theorem is justified by the relevant act. The proven theorem, a piece of knowledge, may thus be called a 'justified judgement'. Instead of the term 'justified judgement', Martin-Löf also uses the term 'evident judgement' (Martin-Löf, 1998, p. 108, 110). From a modern point of view, the term 'evident' suits better the knowledge that results from an act of understanding, because of the English term 'self-evident'. ${ }^{8}$ Within phenomenology, though, the term 'evident' is applied both to immediately and mediately evident judgements. Martin-Löf follows this tradition when he uses the term 'evident judgement' to cover both the results of acts of understanding, the axioms (the immediately evident judgements), and the results of acts of inference, the proven theorems (the mediately evident judgements).

An elucidation of knowledge as justified or evident judgement differs from modern explanations of knowledge in terms of warrant and causality. The constructivist notion of knowledge is an internalist one in the sense that the act of the knowing subject uniquely determines whether that subject has knowledge. It does not imply transparency, though, in the sense that one always knows that one knows; one may not have a clear perception of one's cognitive act. Complete transparancy would involve an infinite regress of cognitive acts-one also would have to know that one knows that one knows, etc.-, which is at least a psychological impossibility. As far as mathematical knowledge is concerned it is the insights of and the inferences made by the mathematical subject that are relevant to the question whether a subject has knowledge. Causality and externalist notions of knowledge seem less relevant as far as mathematical knowledge is concerned. When the constructivist notion of knowledge is extended to non-mathematical cases, the knowledge obtained also needs to be explained as the result of a cognitive act. To take perceptual knowledge as an example, knowledge that this bird has a red breast may be conceived of as the result of an act of

\footnotetext{
7 A comparison of the notions of knowledge, judgemental correctness and the law of excluded middle in Brentano and Martin-Löf can be found in van der Schaar (2004).

8 The term 'evident' as it is used here, in Brentano and in later writings of Martin-Löf, is said of a judgement, and differs from the notion of evidence for a judgement. In an older work from 1987, Martin-Löf speaks of the evidence for a judgement. It is the cognitive act, the proof-act or act of understanding that is the evidence for a judgement (Martin-Löf, 1987, pp. 417-418). The judgement is made evident by the evidence (the cognitive act) for it. Because 'evidence' also means pieces of evidence, I have prevented to use this term.
}

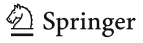


(judgemental) perceiving that this bird has a red breast. Essential to a constructivist explanation of knowledge is that knowledge is the product of an act of the knowing subject. If the knowing subject is confronted with an inconsistency in his judgement products, he should reconsider at least one of his cognitive acts. What he thought was a cognitive act, he may no longer consider being so. The evident judgement is thus fallible (Martin-Löf, 1991, p. 144). In contrast to traditional foundationalism, it is not required that the foundations of knowledge, the judgements that are immediately evident, exhibit an immunity from error. Further, the constructivist explanation of knowledge differs from standard explanations of knowledge in the sense that it does not contain a notion of non-epistemic, absolute truth. The evident judgement may be called 'true' in an epistemic sense, precisely in so far as it is possible to make it evident. Truth in an epistemic sense or judgemental correctness is the evidenceability or justifiability of the judgement (Martin-Löf, 1998, p. 109).

The assertion-candidate expressed by a declarative sentence $S$ can now be explained. In the former section it was shown that the assertion-candidate gives the meaning of the declarative in terms of its assertion-condition. If one follows the explanation of assertion given there, one is entitled to assert that $S$, if and only if one is epistemically justified to assert that $S$, and if one follows Martin-Löf in the explanation of knowledge as justified judgement, then the assertion-candidate expressed by a declarative sentence $S$ can be explained in terms of the knowledge that $S$. The assertion-candidate thus expressed can now be explained by the knowledge one must have in order to be entitled to assert that $S$, which is the knowledge that $S$. For example, one is entitled to assert that the queen (of England) is dressed in pink (on the wedding-day of her first grand-son), if one's judgement is the result of an act of perceiving that the queen is dressed in pink. The assertion-candidate expressed by the declarative 'The queen (of England) is dressed in pink (on the wedding-day of her first grand-son).' is explained in terms of this knowledge.

In a similar way, the account of assertion given in the former section amounts to a knowledge account of assertion. To assert that $S$ is elucidated as to claim that one is epistemically justified to assert/judge that $S$. On the constructivist account of knowledge this means that to assert that $S$ is to claim that one knows that $S$ (not to be confused with asserting that one knows that $S$ ). The knowledge account of assertion just given differs from the one given, for example, by Timothy Williamson. ${ }^{9}$ From a constructivist point of view, one is entitled to assert that $S$ precisely if one's judgement/assertion that $S$ is justified or evident, that is, if it is the result of a cognitive, judgemental act, such as an act of inference or an act of perception. The judgement need not be true in a non-epistemic, absolute sense.

From a constructivist point of view, there are at least two notions of truth that one needs a bearer for. There is epistemic truth, that is, judgemental correctness or evidenceability. The judgement that is the bearer of correctness is a judgementcandidate, which is precisely the same as the assertion-candidate. One may thus ask whether a judgement- or assertion-candidate is correct independently of whether the corresponding judgement is made. The candidate is correct if it is possible to justify it, to make it evident through a cognitive act.

Truth as it functions in the standard explanation of the meaning of a declarative in terms of its truth-condition may be called semantic truth (cf. Sundholm, 2004,

9 A knowledge account of assertion can be found in Moore (1962, p. 277), Sundholm (1999, pp. 120-121) and Williamson (2000), among others. 
p. 437; Sundholm speaks of the different roles of truth). The bearer of semantic truth is the proposition. A proposition is true precisely if a corresponding truth-maker exists. That is, if $A$ is a proposition, the judgement

$A$ is true

can be identified with the judgement

there exists a proof of $A .^{10}$

Such a proof is not an epistemic proof-act, but a proof-object, which is a nonepistemic, mathematical object. In the non-mathematical case, the proof-object may be the red of this robin's breast that makes the proposition that the breast of this robin is red true. It is essential to constructivism that the person who asserts that there exists a proof of $\mathrm{A}$, is entitled to make that assertion, only if he is entitled to make the assertion: a is a proof of $A .{ }^{11} \mathrm{He}$ is allowed to make the existential claim only upon possessing a proof for $A$ (cf. Sundholm, 2004, p. 449). From a constructivist point of view, the judgement $A$ is true is an abbreviation of the judgement $a$ is a proof of $A$.

How can the thesis that the declarative sentence expresses an assertion-candidate be brought into conformity with the constructivist account of proposition, which is said to be the meaning of the declarative? Arend Heyting, who worked within the intuitionist framework of L.E.J. Brouwer, has given a constructivist explanation of the notion of proposition from the thirties onwards. Heyting used a terminology that he (and Oskar Becker) had found in Husserl's Logical Investigations. He explained the proposition as an intention and its truth in terms of the fulfilment of that intention. A construction, which is intended by a mathematical proposition, and which is a proof, a mathematical object, proves or fulfils that proposition. In 1956 Heyting explains the meaning of a proposition in terms of its assertion-condition. A proposition "can be asserted as soon as a construction has been carried out" (Heyting, 1956, p. 98). And the conjunctive proposition " $\mathrm{p} \wedge \mathrm{q}$ can be asserted if and only if $\mathrm{p}$ and $\mathrm{q}$ can be asserted." (Heyting, 1956, p. 97). A conjunctive proposition is then explained in terms of the proofs of each of its conjuncts.

The explanation of proposition in Martin-Löf's writings is obtained by making a distinction between canonical (or direct) and non-canonical (or indirect) proofs, where a non-canonical proof is a method for obtaining a canonical proof. A proposition is explained by laying down how its canonical proofs are formed. And the logical operations are explained in terms of the canonical proofs for the propositions that have the relevant operator as main-connective (cf. Martin-Löf, 1984, p. 12; Sundholm, 1986, p. 485). If the conclusions that we derive were obtained by canonical proofs only, they would all be trivial, that is, they would be deprived of epistemic value. In logic, though, we often derive the truth of a proposition by means of a noncanonical proof. For example, the truth of a conjunctive proposition may be obtained not only by Conjunction Introduction, but also by Disjunction Elimination, that is, by a non-canonical proof. Because of the distinction between canonical and non-canonical proofs it is possible to explain that the conclusions that we derive by the logical rules may have epistemic value (cf. Sundholm, 1986, p. 487).

\footnotetext{
10 The notion of existence that is used in this judgemental form has to be different from the notion of existential quantifier that is present in existential propositions.

11 'a' is a constant naming a proof of $A$.

䡒 Springer
} 
Within the Martin-Löf framework, propositions are identified with sets, that is, sets of their canonical proof-objects. Propositions themselves, though, form a type (in the sense of category), not a set, because we cannot prescribe how the objects of that type (all propositions, including all atomic ones) are formed (cf. Martin-Löf, 1984, 21f.). To understand a category or type is to know when one is entitled to assert that a certain object is an object of this type, and one has to know what it is for two objects to be equal objects of the same type. The proposition $A$ can also be conceived of as a type whose objects are the (canonical and non-canonical) proof-objects of $A .{ }^{12}$ Thus, in order to understand a proposition, that is, to be entitled to make the judgement

$A$ is a proposition,

one must be entitled to make the judgement

a is a proof of $A(\mathrm{a}: A)$,

and one must be entitled to make the judgement

$\mathrm{a}$ and $\mathrm{b}$ are the same proof of $A(\mathrm{a}=\mathrm{b}: A)$,

given that a and $\mathrm{b}$ are proofs of $A$.

Notwithstanding the fact that proof is the central notion in the constructivist meaning-explanation of propositions, the explanation of proposition in terms of proofs need not be interpreted in a constructivist manner, because these proofs are mathematical proof-objects, or, in the non-mathematical case, such as that of perception, perceivable objects like the red of a robin's breast. A realist will conceive of a proofobject a of A as existing independently of a subject's entitlement to assert that a is a proof-object of A. As we have seen above, from a constructivist point of view, one is entitled to make the judgement $A$ is true, which can be identified with the judgment there exists a proof of $A$, only if one is entitled to assert $a$ is a proof-object of $A$, that is, only if the judging person has acquired, constructed a certain proof-object within a proof-act, that is, within a cognitive act. From a constructivist point of view, the nonepistemic notions of proposition and proof-object are thus essentially embedded within a cognitive theory of judgement and proof-act.

In Frege's Begriffsschrift, there is only one judgemental form, $A$ is true ( $A$ 'ist eine Tatsache'), and it thus seems natural to take the part captured by 'is true' as a sign of assertive force (although there is not such a sign in natural language), and $A$ as corresponding to the proposition that is asserted to be true. Type theory is in need of different judgemental forms, though. Not unlike Frege's Begriffsschrift, within type theory, the inference rules do not apply to propositions, but to judgements. Propositions are those entities that the logical connectives operate upon, but as such they cannot function as premises and conclusions, because propositions have the form of a that-clause. Within type theory the introduction rule for conjunction is:

( $A$ is a proposition $B$ is a proposition) $A$ is true $B$ is true $A \wedge B$ is true

The judgements $A$ is a proposition and $B$ is a proposition are presuppositions of the judgements $A$ is true and $B$ is true (cf. Martin-Löf, 1984, p. 44; 1996, lecture 3). A full

12 According to the rules of type-formation, we have a ground type of sets (set: type), and whenever we have a set ( $A$ : set) we may form a new type, namely the type of elements of $A$ (elem $(A)$ : type); and elem $(A)$ can be abbreviated to $A$. 
introduction rule also makes the proof-objects explicit. For example, the proof-object for the conjunction is a pair consisting of a proof-object for $A$ and a proof-object for $B$. As we have seen above, judgements of the form $A$ is true are abbreviations of judgements of the form $a$ is a proof-object of $A$. It is thus judgements of the form $a$ is $a$ proof-object of $A$, or, in a more generalized form, judgements of the form $a$ belongs to type $\alpha$, that is, $a$ is an $\alpha$ (a: $\alpha$ ), which need to be explained in order to understand what a judgement is of the form $A$ is true. Martin-Löf acknowledges four general forms of judgement: ${ }^{13}$

$$
\begin{aligned}
& \mathrm{a}: \alpha \quad \alpha: \text { type } \\
& \mathrm{a}=\mathrm{b}: \alpha \alpha=\beta: \text { type }
\end{aligned}
$$

'Type' can be taken in the sense of set or proposition, for example:

$$
\begin{aligned}
& \text { a :A } A: \text { prop } \\
& \mathrm{a}=\mathrm{b}: A A=B: \text { prop }
\end{aligned}
$$

or in the sense of type as category, for example:

$$
\begin{aligned}
& A: \text { prop prop : type } \\
& A=B: \text { prop prop }=\text { set }: \text { type }
\end{aligned}
$$

These judgemental forms show that the proposition has an important part to play in semantics, but that propositions can answer the question what the meaning is of a declarative sentence only in a partial way. For example, the declarative 'That snow is white is a proposition.' is about a proposition, but it does not express a proposition. (And, not only constructive type theory is in need of such presuppositions.) Not all judgemental forms can be abbreviated to the form $A$ is true. The judgement prop : type (propositions form a type) cannot be abbreviated to this form, because the judgement $A$ is true precisely presupposes the correctness of the judgement that propositions form a type. Again, the declarative 'Propositions form a type.' does not express a proposition. Constructive type theory is in need of an entity that fulfils the role of the meaning of a declarative sentence corresponding to whatever judgemental form, and it is the assertion-candidate that fulfils this role. Propositions also play an important semantic role, because most judgements can be abbreviated to the form $A$ is true. For the corresponding declarative, the proposition $A$ may be called the descriptive meaning. The above-mentioned sentences 'That snow is white is a proposition' and 'Propositions form a type' do not have a descriptive meaning.

Above it was said that the inference rules apply to judgements, but is it judgementproducts or judgement-candidates to which the inference rules apply? Standardly, the inference rules bring us from asserted, that is, known premises to an asserted conclusion, but we also have to account for the fact that the inference rules may apply to assumptions and what is inferred from them. The inference rules thus apply to

13 These four categorical forms of judgements form a special case - a case with zero hypothesis - of hypothetical forms of judgement.

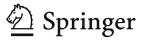


judgement- or assertion-candidates, whether asserted or non-asserted, which is another role for the assertion-candidate not mentioned at the beginning of this section. ${ }^{14}$

The assertion-candidate is also of importance in the context of a hypothetical judgement. Such a judgement may be manifested by the conditional sentence: 'If it rains a lot, then the grave will become moss-grown'. Traditionally, a hypothetical judgement is explained as a connection of two judgements: 'judgement' here means assertioncandidate, for the antecedent 'it rains a lot' is not asserted (or judged). The antecedent is embedded, and thereby loses its assertive force, but keeps the grammatical form of a declarative. The conditional is not an implication (cf. Sundholm, 1998, 185f.). An implication is a proposition $A \rightarrow B$, which relates two propositions $A$ and $B$ by a truth-functional connective. For instance, in a classical framework the meaning of an implicational clause, the implicational proposition, is given by the truth-conditions for the implication in question, its truth-table. Or, within constructivist type theory, an implication is explained by its canonical or direct proof-object, which is obtained by deriving $B$ is true from the non-modal, alethic assumption $A$ is true, that is, the assumption a proof-object of $A$ exists. The logical operators such as implication and conjunction operate upon propositions, not upon assertion-candidates. In contrast, the meaning of the conditional sentence is an assertion-candidate, in which two assertion-candidates are connected. It has the form: if $A$ is true, then $B$ is true, that is, the form of a hypothetical judgement, in type theory: $B$ true ( $A$ true). The assumption that needs to be made in order to prove that a hypothetical judgement is correct is an epistemic, modal assumption that the assertion-candidate $A$ is true is knowable. One assumes that the judgement a proof-object of $A$ exists is knowable, that is, that a proof-object of $A$ can be found (cf. Sundholm, 2004, 451f.).

The assertion-candidate also fulfils the role of being the content of a judgement or assertion. One might also say that the proposition is the (descriptive) content of an act of assertion, in the sense that the proposition is the content that is asserted to be true, but this makes sense only for judgements of the form $A$ is true. That the content of the assertion is the assertion-candidate in the first place, and not the proposition, will become clear when the question is asked what the content is of the other acts in the next section on mood and force.

\section{A theory of mood}

Is it possible to give a theory of mood that is able to take into account all the points mentioned at the end of section 2? A semantics is needed that can account for what is common to the sentences in the different moods, and that can also account for the difference between each of the sentence types. An utterance of an interrogative sentence is standardly (without counter-indications) understood as being used for asking a question. In analogy with the semantics given for the declarative, one may say that the meaning of the interrogative sentence is the question-candidate (or interrogabile). ${ }^{15}$ The meaning of the sentence 'Is it snowing?' is the question-candidate is it snowing. The question-candidate for such closed questions is not individuated in

\footnotetext{
14 According to Sundholm, assumptions get the form of a hypothetical judgement If $A$ is true, then $A$ is true, or $A$ true (A true) (Sundholm, 2002, p. 576). Because assumptions have thus disappeared, the inference rules can be said to apply to assertion-products only.

15 The Paris logician Adam of Balsham introduced this notion in his Ars disserendi (1132): "Adam introduced the term interrogabile for that which is capable of being asked, even if it is not actually
} 
terms of question-conditions, but by what count as possible answers to the question that is (standardly) asked by means of an utterance of the corresponding interrogative sentence. The answers have declarative form, and they need not be actual answers. They are therefore assertion-candidates. Possible answers to the question 'Is it snowing?' are the assertion-candidates it is snowing and it is not snowing. Candidates for open questions, such as who did it, are explained by more generic answerhoodconditions, similarly consisting of assertion-candidates (someone did it). The meaning of an imperative sentence, 'Close the door!', can be explained by the command that the sentence is standardly understood to have, and is a command-candidate. The command-candidate can be individuated in terms of the obedience-condition of the corresponding command. Such obedience-conditions can be expressed by declarative sentences ('The door is closed.'), and are thus assertion-candidates. Perhaps one should account for the fact that the command is directed to a certain person, for example, John. The obedience-condition can then be expressed by the declarative 'John has closed the door.' In most languages there is also an optative mood, and a corresponding optative sentence, for example 'May it snow at Christmas.' The meaning of the optative is the wish-candidate may it snow at Christmas, which is individuated in terms of what counts as a fulfilment of the corresponding wish-act. The possible fulfilment can be expressed by the declarative 'It snows at Christmas.', and is thus an assertion-candidate. In general, the meanings of the different sentence types can all be explained in terms of their specific relations to assertion-candidates, and those specific relations are determined by the speech act that an utterance of such a sentence is standardly understood to have.

It is contingent how many sentence types or moods a language has, and thus how many candidate notions it needs. It is sometimes said that language has its origin in a language-game consisting of nothing but imperative sentences. In such a form of life one would also need the possibility to determine whether a command is obeyed, and one would thus be in need of the assertion-candidate. The declarative mood is essential to language. Below, something will be said about those types of force for which the English language does not have a special mood.

In all these cases the product of the act, the question asked, the command given, is to be distinguished from the respective candidate notions. According to Frege, the Bedeutung of the that-clause in the sentence 'He commanded that we should retreat.' is a command (Frege, 1892, pp. 38-39). In analogy with Frege's analysis of ordinary oratio obliqua sentences, it may be said that the Sinn of an imperative sentence is a command. Because such a 'command' is needed also when nothing is actually commanded ('No, he did not command that we should retreat'), the 'command' cannot be the product of an act of commanding: it has to be a command-candidate. Frege, though, did not make a clear distinction between a given command (the product) and the command-candidate. Frege's thesis that declarative and imperative sentences differ in sense (Sinn) is not 'definitely wrong', as Dummett thought (Dummett, 1973, p. 307). Contrary to what Dummett has said, it is possible to speak of "commands which have not been given" (Dummett, 1973, p. 303).

Footnote 15 continued

asked," (Nuchelmans, 1973, p. 169). The notion Frageverhalt, introduced by Johannes Daubert, a Munich phenomenologist, is similar to that of question-candidate. Cf. Schuhmann and Smith (1987, p. 367). The explanation given on page 368: "a peculiar ideal sense-formation: the wish itself, command, question, assertion, declaration itself ... which "becomes an event" in the act" perfectly suits the candidate notions. Daubert's Erkenntnisverhalt corresponds to the assertion-candidate.

Springer 
There is an important argument against a theory of meaning that includes mood. If one has explained what a word means in a declarative, one has not explained what it means in an imperative or an interrogative sentence (cf. Dummett, 1973, pp. 307-308). The criticism does not apply, though, because the meanings of the different types of sentences, the different candidates, are all explained in terms of the assertion-candidate.

The content of an act of questioning that is made known by means of an utterance of the sentence 'Is the door closed?' is the question-candidate is the door closed. ${ }^{16}$ The content of the command 'Close the door!' is the command-candidate close the door. The content of these acts are thus distinguished from the proposition that the door is closed. ${ }^{17}$ The proposition may be called the descriptive content; the different candidate notions may have the same descriptive content.

The thesis that the different sentence types express different types of candidates can be elucidated by the Stoic notion of lekton. An example of a complete lekton (i.e., a complete meaning) is an axioma, which is explained as thought expressed in a speech act of assertion (Nuchelmans, 1973, p. 73). The antecedent and consequent of a conditional is called axioma or axioma kata dynamin ('potential statement') (idem, 61). Other lekta include (candidates for) questions, commands and oaths. They are the meaning-entities by which we perform our speech acts (in so far as the meaning is embodied in language). ${ }^{18}$

What can be the common core of these different sentence types? One may follow Hare (1970) in his theory of mood and his corresponding analysis of speech acts. Hare takes the phrastic to be that part of a sentence that expresses the descriptive content (the proposition). A speech act is represented by adding to the phrastic a sign of mood, the tropic, and a sign of subscription, the neustic. The sign of mood shows what type of speech act we normally, by convention, make with a sentence in this mood. A sign of subscription, finally, indicates that the speech act in question has been performed. We thus need a general subscription sign. In the Begriffsschrift, Frege analyzed what is now called the assertion-sign as consisting of two parts: the horizontal, a sign showing that the content is judgeable, and the vertical, a sign showing that the content is asserted. Applying the distinction to the theory presented here, we may take the horizontal '- ' as corresponding to the is true part of the assertion-candidate, and the vertical as a sign showing that the content $A$ is true is asserted. I will use Frege's vertical as sign of subscription, thus generalizing it beyond the judgement. I double the sign for visual reasons, thus obtaining ' $\|$ ' as a general sign of subscription. Taking '-', '?' and '!' as signs of the meaning of mood, not of (actual) force, an act of assertion, an act of questioning and an act of command can now be analyzed, respectively, as:

$$
\begin{aligned}
& \|-A \\
& \| ? A \\
& \| ! A
\end{aligned}
$$

The common core is thus the proposition $A$.

\footnotetext{
16 Nuel Belnap (1990) introduced the appropriate term 'interrogative content' for a notion similar to the question-candidate.

17 The content of the act can thus also not be identified with the identical, intentional object that the door is closed to which the different acts are directed, which Husserl called a 'state of affairs' in the fifth Logical Investigation (Husserl, 1901, V, Sect. 17 and Sect. 28).

18 "Offenbar kann man mit einem vollständigen Lekton einen illokutionären Sprechakt vollziehen," (Hülser, 1992, p. 26); cf. Nuchelmans (1973, p. 63, 67).
} 
This analysis of speech acts is more complicated than the traditional analysis, which identifies mood with force. Erik Stenius, who identifies mood with force ('function', Stenius, 1967, p. 256), takes 'I' to be a sign of the typical function of the declarative ('Indicative') mood, and ' $(p)$ ' to represent (the linguistic counterpart of) the proposition. The analysis of the declarative, including its force, is thus: I $(p)$. Similarly, the analysis of an interrogative, including force, is, according to Stenius, ? $(p)$. Stenius' analysis of 'mood' does not essentially differ from Searle's early analysis of speech acts. According to the analysis of speech acts given above, the speech act should be analyzed into candidate and subscription, whereupon the candidate may be analyzed into proposition and the meaning of mood.

The analysis given above represents the relation between proposition, mood and typical force in a perspicuous manner, but it does not answer all questions concerning the relation between mood and force, and that between mood and the common core. One may also wonder how all the different types of speech acts - to promise, to warn, to apologize, etc. - are analyzed. There seems to be an infinite amount of types of speech acts, whereas there is only a small amount of types of mood. This point will be dealt with below under the topic of deviant uses of mood.

The analysis is somewhat misleading as far as the conceptual order is concerned. Above the assertion-candidate is explained in terms of the speech act of assertion, and as far as the conceptual order is concerned one should start with a sign of the speech act of assertion, and add a sign that functions as a modifying operator by which the assertive force is cancelled and the assertion-candidate is obtained. When $S$ is used to represent the speech act of assertion, and when the round brackets ( ) are used as modifiers, the assertion-candidate can be represented as: $(S)$.

Another problem with the analysis given above is that not all assertions and questions have a proposition as common core. The questions 'Is $A$ a proposition?' and 'Do propositions form a type?' and the assertions ' $A$ is a proposition.' and 'Propositions form a type.' do not have a descriptive content, and can thus not be analyzed as, respectively,

$$
\| ? A \text { and } \|-A \text {. }
$$

Finally, it is not clear how open questions can be represented this way, because they do not share a proposition as common core with the other speech acts. The open question, though, does have something in common with the other speech acts: they all have the assertion-candidate as part of their explanation. The assertion 'John committed the crime' is a possible answer to the open question 'Who committed the crime?', because it conforms to the genereric answerhood-conditions that are determined by the open question-candidate.

Possibly, we may solve these problems by giving a more prominent place to the assertion-candidate. Perhaps Davidson had a point, when he defended the thesis that declarative sentences form the common core of the different sentence types. The semantic counterpart of the declarative, the assertion-candidate, may after all be the common core of the different moods.

A theory of mood need not only be complete, it also needs to explain our intuition that there is a special relation between mood and corresponding force. Above, it is shown that there is such a relation between mood and potential force. Further, our theory of mood needs to explain the deviant uses of the moods mentioned at the end of Sect. 2. How can one explain that a command to open the door may be given through an utterance of the declarative sentence 'The door is closed.', or through an 
utterance of the interrogative sentence 'Could you open the door?'. In such cases we do use the utterance of the declarative and that of the interrogative, respectively, with assertive and interrogative force. Besides, the context contains non-conventional cues by which one can determine that a command is conversationally implicated (Grice, 1989, p. 26; cf. Pendlebury, 1986, p. 369). If an utterance of 'The door is closed.' is used for a command, Grice's maxim of quantity (Be as informative as is required) is exploited: both speaker and hearer can see that the door is closed. A free-standing utterance of the declarative 'I promise to give it back.', though, cannot be understood as having assertive force. The utterance merely counts as a speech act of promising. The 'I promise' sentence cannot be called 'correct' or 'incorrect' in the way 'The door is closed' can be called so. The assertive force is cancelled by a special use of the performative verb 'to promise', which is able to fulfil its performative, and thus assertion-cancelling, function in so far as it is used in the first person present. A similar solution one must give for other Austinian cases: 'I congratulate you', 'I apologize'. An account of these performatives goes beyond a theory of mood, because (the English) language does not have a special mood for promising or apologizing.

How can we explain that one may use an utterance of the sentence 'The door is closed' not for a making a full assertion, but for a weaker type of speech act, which we may call assent? In the case of assent one merely expresses one's opinion without claiming that one knows. In such a case, there should be an indication that the speaker is not making a full assertion, for example, by adding in parenthesis 'I think'. The speech act of assertion is thus modified into a weaker type of act. A command may be modified into a weaker type of speech act, a request, by adding 'please' to the imperative sentence, or by the knowledge common to speaker and hearer that the speaker does not have any authority in relation to the person to whom he is directing his speech act.

A stage-setting also modifies the standard use of the uttered sentences. Utterances of free-standing declaratives on stage are not taken as uttered with assertive force. Frege was right when he called such an utterance a 'sham assertion' (Scheinbehauptung, Frege, 1918, p. 63); 'sham' is a modifier. To paraphrase Austin, these are the etiolations of language, parasitic upon its normal use (Austin, 1962, p. 22). According to Dummett, the assertive force, although 'transposed', cannot be cancelled in such cases because the audience must be able to tell the difference between the character's making an assertion and his asking a question (Dummett, 1993, p. 212). But on stage the assertive force is cancelled. The audience is able to tell the difference, because the character is using a declarative that would have assertive force in a similar real-life situation.

In the case of examples and quotations the modification is complete. The blackboard is a sign that the occurrence of the declarative is not used to make an assertion. In written language we use quotation-marks, which function as modifiers, just as in speech the phrase 'John says that' cancels the assertive force of the declarative that follows.

Finally, not only declarative sentences may be used as subordinate clauses, also imperative and optative sentences may be used that way: 'Write a paper or have an oral examination.' Only the free-standing sentence can have imperative force. Like the word 'if', 'or' cancels the imperative force of each of the subordinate clauses. Each of the subordinate clauses expresses merely a command-candidate. 
Whereas pragmatics deals with the actual force of the utterance of a sentence and with its different deviant uses, the candidate notions belong to semantics. The candidate notions are explained by means of the pragmatic notion of force, and may therefore be called the pragmatic meaning, in contrast to the descriptive meaning of the sentence, the proposition (cf. Recanati, 1987). What is relevant to determine the pragmatic meaning of a sentence is merely the force that a free-standing utterance of that sentence would have in a typical situation, that is, what is relevant is the potential force of the sentence. The pragmatic meaning of a sentence can be determined independently of the use that is made of the sentence, and is therefore part of semantics, not of pragmatics. Mood is a characteristic of sentences independent of their use, and is thus present in free-standing sentences as well as in subordinate clauses. Actual force is a characteristic of utterances of free-standing sentences only; it cannot be determined independently of the use that is made of a sentence. It is not true, as Dummett has said, that a theory of actual force belongs to a theory of meaning; a theory of actual force belongs to pragmatics.

Others have presented a theory of meaning for mood distinguished from a theory of force, for example, Alston (2000), Recanati (1987) and Pendlebury (1986). Alston identifies sentence meaning with speech act ('illocutionary act') potential, and thus comes close to the explanation given above. Alston would probably accuse the theory presented here as having 'the assertoric bias' (Alston, 2000, p. 303), because the notion of assertion-candidate is used to explain the other candidate notions. The order in which the concepts of assertion and assertion-candidate are explained is also different from the one given by these other writers. Recanati, for example, says that "a true assertion is really a kind of stage assertion with an additional element." (Recanati, 1987, p. 266). In the order of explanation I have proposed, assertion on stage is to be explained in terms of the prior notion of (full) assertion together with the modifying context of the stage-setting which cancels the assertive force. Finally, the constructivist account of mood explains the meaning of mood not only in pragmatic terms, but also in cognitive terms. Because the speech act of assertion is a cognitive notion, the assertion-candidate, which is explained in terms of assertion, is thereby explained in cognitive terms.

\section{Conclusion}

The assertion-candidate expressed by a declarative sentence $S$ is explained by the knowledge one needs to have in order to be entitled to assert that $S$, and this is the knowledge that $S$. It is thus explained in cognitive terms, and in terms of the speech act of assertion that is standardly made by means of an utterance of the corresponding declarative. The meaning of mood is thus not explained in terms of actual force (contra Dummett), but in terms of potential force. Such a semantics of mood is in need of a distinction between standard and non-standard uses of the different sentence types. An account of the distinction is possible (contra Davidson): without counterindications, an utterance of a free-standing declarative is understood as having assertive force. Analogous to the explanation of the meaning of the declarative, an explanation for the meaning of other sentence types is given. The other candidate notions can ultimately be explained in terms of their specific relations to the assertioncandidates. Within constructive type theory the semantic notion of proposition needs to be embedded in a semantics that has an explanation for mood. The Martin-Löf 
framework is also used to show the philosophical relevance of the assertion-candidate. Whereas the proposition is needed as descriptive meaning of the declarative and as bearer of semantic truth, the assertion-candidate fulfils the office of being the pragmatic meaning of the declarative sentence, the content of judgement and assertion and the bearer of epistemic truth.

Acknowledgements I thank Per Martin-Löf, Göran Sundholm, Igor Douven, the audience in Paris at Institut d'Histoire et de Philosophie des Sciences et des Techniques (IHPST) and Institut Jean Nicod and the two anonymous referees for comments on a former version of this paper. I want to thank the IHPST for their hospitality during my sabbatical leave in the fall of 2005.

\section{References}

Alston, W. P. (2000). Illocutionary acts and sentence meaning. Ithaca, London: Cornell University Press.

Austin, J. L. (1962). How to do things with words. 1984. Oxford, New York: Oxford University Press.

Belnap, N. (1990). Declaratives are not enough. Philosophical Studies, 59, 1-30.

Davidson, D. (1979). Moods and performances. In his Inquiries into Truth and Interpretation, 109-121. Oxford: Clarendon Press. 1984. Originally in A. Margalit (Ed.), Meaning and use. Dordrecht: Reidel, 1979.

Davidson, D. (1984). Communication and convention. In his Inquiries into Truth and Interpretation, 265-280. Oxford: Clarendon Press.

Dummett, M. (1973). Assertion. Chapter 10 in his Frege: Philosophy of Language, sec. ed., 295-363. London: Duckworth, 1992.

Dummett, M. (1976). What is a theory of meaning (II). In his The Seas of Language, 34-93. Oxford: Clarendon Press. 1993. Originally in G. Evans and J. McDowell (Eds.), Truth and meaning. Oxford: Clarendon Press, 1976.

Dummett, M. (1993). Mood, force, and convention. In his The Seas of Language, 202-223. Oxford: Clarendon Press, 1993.

Frege, G. (1892). Über Sinn und Bedeutung. Zeitschrift für Philosophie und philosophische Kritik, $100,25-50$.

Frege, G. (1918). Der Gedanke. Beiträge zur Philosophie des deutschen Idealismus, 1, 58-77.

Grice, P. (1989). Studies in the way of words. Cambridge, Ms., London: Harvard University Press.

Hare, R. M. (1970). Meaning and speech acts. The Philosophical Review, 79, 3-24.

Heyting, A. (1956). Intuitionism. Amsterdam: North-Holland Publishing Company.

Hinzen, W. (2000). Anti-realist semantics. Erkenntnis, 52, 281-311.

Huddleston, R., \& Pullum, G. K. (2002). The Cambridge grammar of the english language. Cambrige: University Press.

Hülser, K. (1992). Stoische Sprachphilosophie. In Sprachphilosophie, 17-34. Berlin, New York: Walter de Gruyter.

Husserl, E. (1901). Logische Untersuchungen, Hua XVIII, E. Holenstein (Ed.). (The Hague: Martinus Nijhoff, 1975); and Hua XIX, U. Panzer (Ed.). (The Hague, Boston, Lancaster: Martinus Nijhoff, 1984.)

Martin-Löf, P. (1984). Intuitionistic type theory. Naples: Bibliopolis.

Martin-Löf, P. (1987). Truth of a proposition, evidence of a judgement, validity of a proof. Synthese, $73,407-420$.

Martin-Löf, P. (1991). A path from logic to metaphysics. In Atti del Congresso Nuovi problemi della logica e della filosofia della scienza, Vol. II, 141-149. Bologna: CLUEB.

Martin-Löf, P. (1996). On the meanings of the logical constants and the justification of the logical laws. Nordic Journal of Philosophical Logic, 1,11-61. (Originally presented at 1983.)

Martin-Löf, P. (1998). Truth and knowability: On the principles C and K of Michael Dummett. In H. G. Dales, \& G. Oliveri (Eds.), Truth in Mathematics, 105-114. Oxford: Clarendon Press.

Moore, G. E. (1962). Commonplace book: 1919-1953. London: Allen and Unwin.

Nuchelmans, G. (1973). Theories of the proposition. Amsterdam, London: North-Holland Publishing Company.

Nuchelmans, G. (1980). Late-scholastic and humanist theories of the proposition. Amsterdam: North Holland. 
Pendlebury, M. (1986). Against the power of force: Reflections on the meaning of mood. Mind, 95, 361-372.

Ranta, A. (1994). Type-theoretical grammar. Oxford: Clarendon Press.

Recanati, F. (1987). Meaning and force. Cambridge: University Press.

van der Schaar, M. (2003). Opinion, assertion, knowledge: Three epistemic modalities. In T. Childers, \& O. Majer (Eds.), The Logical Yearbook 2002 (pp. 259-268). Prague: Filosofia.

van der Schaar, M. (2004). Brentano on logic, truth and evidence. Brentano Studien, 10(2002 / 2003), $119-150$.

van der Schaar, M. (2006). On the ambiguities of the term judgement. An evaluation of Twardowski's distinction between action and product. In A. Chrudzimski, \& D. Lukasiewicz (Eds.), Actions, Products and Things: Brentano and Polish Philosophy (pp. 35-53). Frankfurt: Ontos Verlag.

Schuhmann, K., \& Smith, B. (1987). Questions: An essay in Daubertian phenomenology. Philosophy and Phenomenological Research, 47, 353-384.

Siegel, H. (2004). Rationality and judgment. Metaphilosophy, 35, 597-613.

Stenius, E. (1960). Wittgenstein's Tractatus. Oxford: Blackwell.

Stenius, E. (1967). Mood and Language-Game. Synthese, 17, 254-274.

Stepanians, M.S. (1998). Frege und Husserl über Urteilen und Denken. Paderborn: Schöningh.

Sundholm, G. (1986). Proof theory and meaning. In D. Gabbay, \& F. Guenthner (Eds.), Handbook of philosophical logic (Vol. III, pp. 471-506). Dordrecht: Reidel.

Sundholm, G. (1997). Implicit epistemic aspects of constructive logic. Journal of Logic, Language, and Information, 6, 191-212.

Sundholm, G. (1998). Inference, consequence, implication: A constructivist's perspective. Philosophia Mathematica, 6, 178-194.

Sundholm, G. (1999). MacColl on judgement and inference. Nordic Journal of Philosophical Logic, $3,119-132$.

Sundholm, G. (2002). A century of inference: 1847-1936. In P. Gärdenfors, J. Wolenski, \& K. Kijania-Placek (Eds.), In the scope of logic, methodology and philosophy of science (Vol. II, pp. 565-580). Dordrecht: Kluwer.

Sundholm, G. (2004). Antirealism and the roles of truth. In I. Niniluoto, M.Sintonen, \& J. Wolenski (Eds.), Handbook of epistemology (pp. 437-466). Dordrecht: Kluwer.

Williamson, T. (2000). Knowledge and its limits. Oxford: Clarendon Press. 